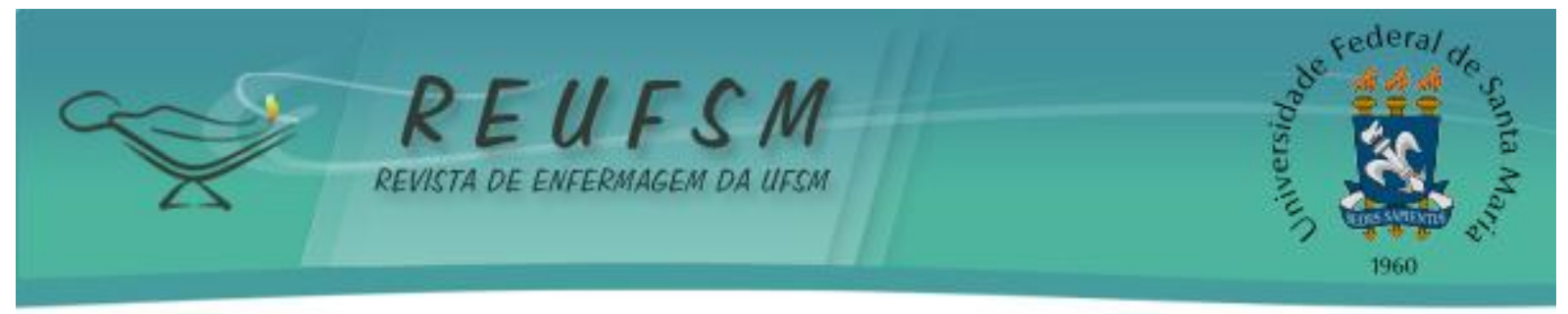

ARTIGO ORIGINAL

\title{
SISTEMATIZAÇÃO DA ASSISTÊNCIA DE ENFERMAGEM NO PERÍODO PERIOPERATÓRIO: VISÃO DA EQUIPE DE ENFERMAGEM
}

\section{SYSTEMATIZATION OF NURSING CARE IN THE PERIOPERATIVE PERIOD: OVERVIEW OF THE NURSING TEAM}

SISTEMATIZACIÓN DE LA ATENCIÓN DE ENFERMERÍA EN EL PERÍODO PERIOPERATORIO: VISIÓN DEL EQUIPO DE ENFERMERÍA

Edlamar Kátia Adamy ${ }^{1}$ Maiara Tosatti ${ }^{2}$

RESUMO: Objetivo: avaliar a implantação da Sistematização da Assistência de Enfermagem (SAE) no período perioperatório de um Hospital do Oeste de Santa Catarina sob a visão da equipe de enfermagem. Método: trata-se de um estudo qualitativo, descritivo realizado por meio de oito entrevistas estruturadas aos técnicos de enfermagem e enfermeiros dos turnos matutino, vespertino e noturno do setor de internamento cirúrgico e Centro Cirúrgico. Resultados: os resultados sinalizam as dificuldades encontradas para a implantação da SAE, entre elas destacaram-se: a falta de comprometimento coletivo, implantação repentina, sem capacitação adequada da equipe de enfermagem, ausência de um sistema informatizado para facilitar o processo de trabalho e déficit no dimensionamento de pessoal. Conclusões: cabe à instituição viabilizar estratégias que culminem para uma SAE efetiva e com resultados mais satisfatórios.

Descritores: Assistência perioperatória; Processos de enfermagem; Avaliação em enfermagem.

ABSTRACT: Objective: aimed to evaluate the implementation of the Nursing Care Systematization (NCS) in the perioperative period of a Hospital of the West of Santa Catarina in the vision of the nursing team. Method: this is a qualitative study descriptive study using structured interviews with eight technicians and nurses of the morning, afternoon and evening sector surgical hospital and surgical center. Results: the results indicate the difficulties encountered in the implementation of the NCS, between it stood out: the lack of collective commitment, sudden deployment without adequate training of nursing staff, lack of a computerized system to facilitate the work process and a deficit in scaling personnel. Conclusion: it is the institution viable strategies that leadto an effective and NCS with more satisfactory results.

Descriptors: Perioperative care; Nursing process; Nursing assessment.

RESUMEN: Objetivo: evaluar la implementación de la Sistematización de la Asistencia de Enfermería (SAE) en el período perioperatorio de un Hospital del Oeste de Santa Catarina, en la visión del equipo de enfermería. Método: se trata de un estudio cualitativo, descriptivo, através de entrevistas estructuradas con ocho enfermeras y técnicos del sector de la mañana, tarde y noche del hospital quirúrgico y centro quirúrgico. Resultados: los resultados indican las dificultades encontradas en la aplicación de la NCS, entre los que destacaron: la falta de compromiso colectivo, la implantación repentina, sin una formación adecuada del personal de enfermería, la falta de un sistema informatizado para facilitar el proceso de trabajo y un déficit en la ampliación personal. Conclusión: se

\footnotetext{
${ }^{1}$ Enfermeira, Mestre em Saúde Coletiva pela Universidade do Oeste de Santa Catarina (UNOESC), Professora Assistente da Universidade do Estado de Santa Catarina (UDESC). Membro do Grupo de Pesquisa Enfermagem, Cuidado Humano e Processo Saúde-adoecimento. E-mail: katiadamy@hotmail.com

${ }^{2}$ Enfermeira, egressa da Universidade do Estado de Santa Catarina (UDESC). E-mail: maitosatti@hotmail.com
} 


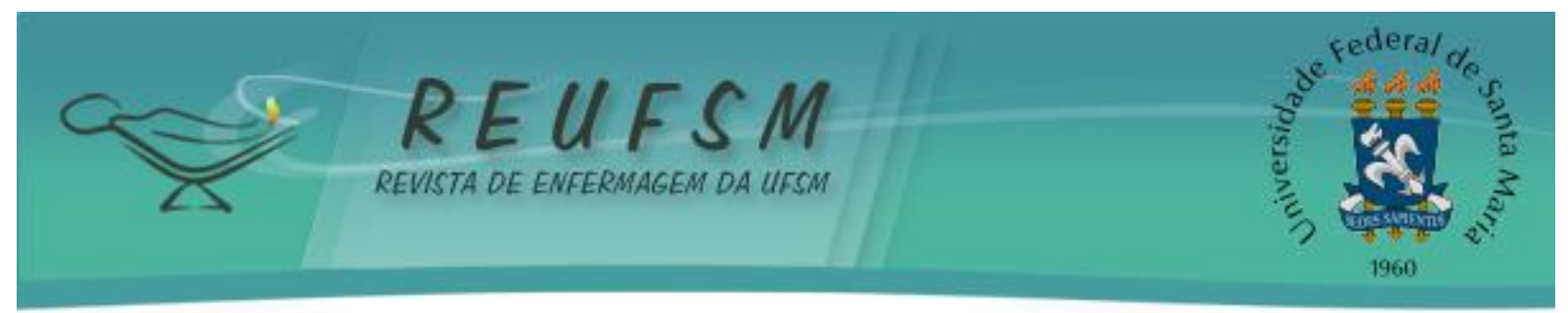

trata de la institución viabilizar estrategias que conduzcan para una SAE efectiva y con resultados más satisfactorios.

Descriptores: El manejo perioperatorio; Proceso de enfermería; Enfermería evaluación.

\section{INTRODUÇÃO}

Através dos tempos, a enfermagem passou por diferentes períodos na sua evolução até a consolidação da enfermagem moderna com a contribuição de Florence Nightingale. As práticas do cuidado passam da responsabilidade da família aos sacerdotes, religiosas, feiticeiros, místicos e médicos para o cuidado da enfermagem leiga no período medieval e pós medieval. Em 1860, Florence Nightingale inicia a prática da enfermagem baseada em conhecimentos principiando a solidificação da cientificidade dos cuidados de enfermagem. ${ }^{1-2}$

A partir do ano de 1950, várias teoristas como Hildegard E. Peplau, Virginia Henderson, Faye Glenn Abdellah e Dorathea E. Johnson buscaram entender a verdadeira necessidade do paciente e o que o enfermeiro poderia fazer a respeito. Então criaram os diagnósticos de enfermagem (DE) que facilitariam o trabalho e melhoraria a assistência ao paciente. $^{2}$

Essas mudanças culminaram para uma prática de enfermagem mais organizada e a implantação da Sistematização da Assistência de Enfermagem (SAE) como um instrumento de trabalho do enfermeiro. A SAE busca promover uma assistência holística, individualizada e mais qualificada ao paciente consolidando o comprometimento e a responsabilidade do profissional perante os pacientes, familiares e comunidade. Neste sentido, proporciona ações tanto na área assistencial quanto administrativa, o que facilita a organização do trabalho e dos serviços da equipe de enfermagem.

O Conselho Federal de Enfermagem - COFEN, através da Resolução n 358 de 15 de outubro de 2009, determina que a implementação da SAE deva ocorrer em toda instituição de saúde, pública e privada, sendo que cabe privativamente ao enfermeiro realizar todas as etapas da SAE. A presente resolução organiza o trabalho profissional quanto ao método, pessoal e instrumentos, tornando possível a operacionalização do Processo de Enfermagem (PE) descrito em cinco etapas: coleta de dados, diagnóstico de enfermagem, planejamento de enfermagem, implementação e avaliação de enfermagem. ${ }^{3}$

Para atender as demandas da prática profissional, o enfermeiro busca executar as atividades de forma humanística, contemplando as necessidades assistenciais e de gerenciamento, o que levou a enfermeira Wanda de Aguiar Horta, a partir do ano de 1979, iniciar com o PE, uma SAE designada para o Centro Cirúrgico (CC), denominada Sistematização da Assistência de Enfermagem Perioperatória (SAEP). ${ }^{4}$

A evolução cirúrgica e novas descobertas de tecnologias ajudaram a enfermagem avançar na assistência perioperatória. Primeiramente a enfermagem era essencialmente instrumental, realizava a limpeza de materiais e auxiliava a equipe médica. Com o passar do tempo, o enfermeiro acabou percebendo a importância da assistência ao paciente no CC. $^{4}$

A dificuldade encontrada pelos enfermeiros em realizar a SAEP se relaciona à equipe administrativa, que muitas vezes não entende a importância do enfermeiro na assistência ao paciente cirúrgico, direcionando cada vez mais a atuação do enfermeiro nas atividades administrativas. ${ }^{5}$

No Brasil, um grupo de enfermeiros de São Paulo identificou a escassez de estudos e pesquisas científicas em relação à assistência no CC, e em 1982 criaram o Grupo de Estudo em CC e Centro de Material (GECC). Após nove anos, visualizaram a potencialidade 


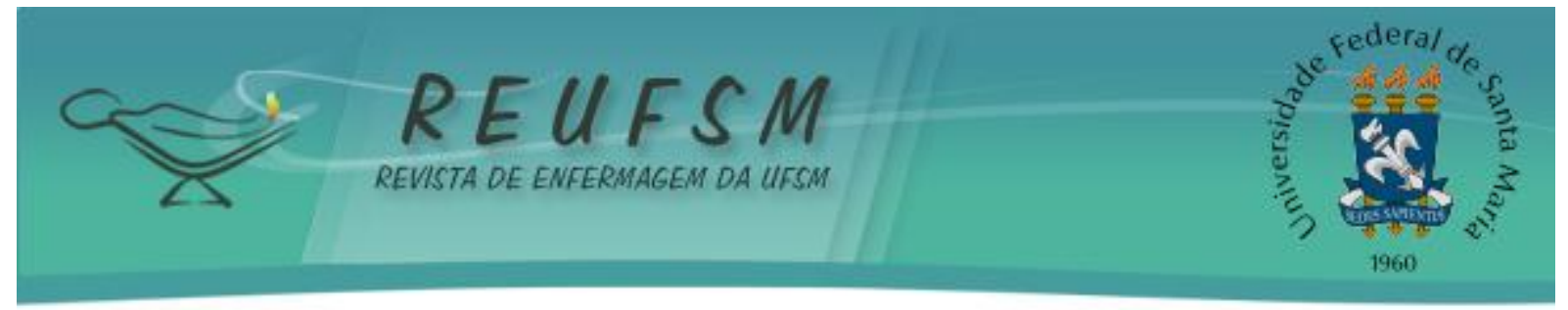

do grupo e em 1991 transformaram o GECC na Sociedade Brasileira de Enfermeiros de Centro Cirúrgico (SOBECC). ${ }^{6}$

A sistematização perioperatória tem por objetivos, proporcionar uma assistência integral e individualizada, ajudando o paciente e a família conhecer e compreender o procedimento que será realizado a fim de tranquilizá-los e tentar diminuir os riscos que um procedimento pode oferecer. Preconiza que a visita pré e pós-operatória sejam a melhor ferramenta para manter a assistência de forma qualificada dando continuidade à sistematização. ${ }^{7}$

"A SAEP traz como benefícios a diminuição do tempo para resultados diagnósticos e tratamento dos problemas de saúde potenciais ou vivenciais, reduzindo a incidência e a duração da permanência dos clientes no Hospital". 8:85

Com a implantação da SAEP, a enfermagem produz uma assistência planejada ao paciente, atendendo todas as necessidades básicas do mesmo e consequentemente 0 serviço da enfermagem se torna mais organizado. ${ }^{7}$

0 interesse pelo presente estudo surgiu durante as atividades teórico práticas, corroboradas pelos questionamentos oriundos dos profissionais do hospital em estudo e que estão relacionados ao processo de implantação e implementação da SAE e das facilidades e dificuldades dos profissionais de enfermagem em efetivar o PE. Assim, estabeleceu-se para este estudo o objetivo de analisar a percepção da equipe de enfermagem sobre a SAE no período perioperatório. Os resultados desta investigação possibilitam intervenções que culminam para a correção dos déficits na busca da qualidade assistencial e da valorização e autonomia do trabalho em enfermagem.

\section{MÉTODO}

Trata-se de um estudo qualitativo, descritivo, desenvolvido em um Hospital Cooperado no Oeste de Santa Catarina, que atende a demanda de média e alta complexidade, com atendimento diário de aproximadamente 55 pacientes no setor de internamento de pacientes cirúrgicos, denominado setor B e no CC, únicos setores que atendem o paciente no período perioperatório. Para atender a esta demanda, o hospital conta com sete enfermeiros e 54 técnicos de enfermagem.

A seleção dos sujeitos da pesquisa ocorreu com convite à equipe de enfermagem, de forma a incluir: enfermeiros e técnicos de enfermagem do setor B e do CC do hospital em estudo e que desenvolvem suas atividades nos turnos: matutino, vespertino ou noturno. Foram excluídos os sujeitos que se recusaram a participar da pesquisa, em férias, folga, afastados ou licenciados no momento da realização das entrevistas. Dos profissionais que se adequavam aos critérios, foram entrevistados quatro técnicos de enfermagem e quatro enfermeiros, num total de oito sujeitos. Os profissionais, que se recusaram a participar do estudo, justificaram a desistência pelo pouco entendimento sobre o que é a SAE, fato que nos leva a refletir.

Os dados foram coletados por meio de entrevista estruturada no mês de maio de 2011 e seguiram um roteiro de perguntas que foram categorizadas em sete itens a serem avaliados, a fim de identificar as potencialidades, dificuldades e questões pertinentes à implantação da SAE. As entrevistas foram realizadas individualmente na própria instituição em horários previamente agendados, gravadas em aparelho eletrônico (MP3) e posteriormente transcritas para análise. As informações obtidas neste estudo foram trabalhadas a partir da técnica de análise de conteúdo. ${ }^{9}$

Para preservar o anonimato dos sujeitos da pesquisa, os mesmos foram identificados pelas iniciais $E$ para enfermeiro e $T$ para técnicos de enfermagem, seguidos de números naturais e em ordem crescente de acordo com a realização das entrevistas. 


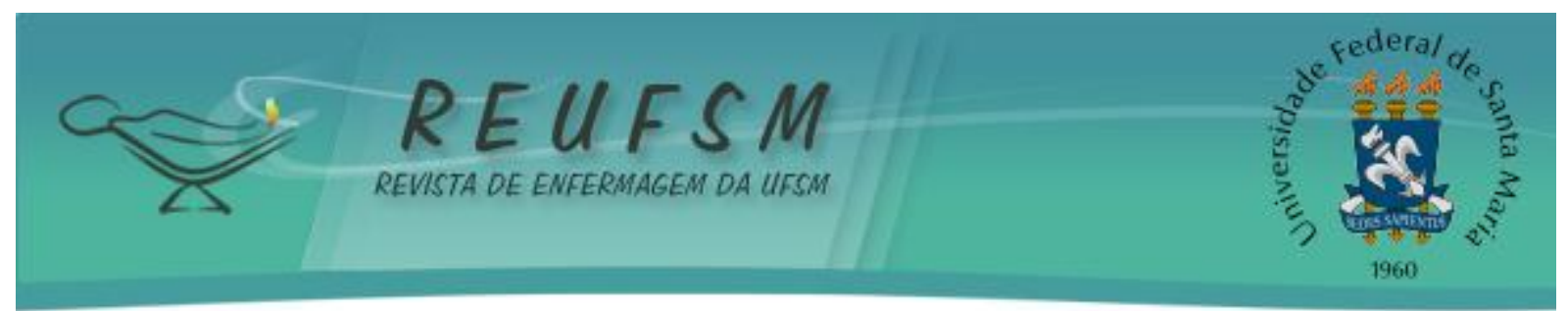

Os sujeitos da pesquisa declararam sua participação no estudo mediante a assinatura do Termo de Consentimento Livre e Esclarecido. 0 presente estudo foi submetido ao Comitê de Ética em pesquisa da Universidade do Estado de Santa CatarinaUDESC, sob número 20/2011 e obedece a resolução 196/1996 do Conselho Nacional de Saúde e os dados da pesquisa serão arquivados nas dependências da universidade.

\section{RESULTADOS E DISCUSSÕES}

A SAE foi implantada em todos os setores do hospital, a partir das exigências para a avaliação da Organização Nacional de Acreditação (ONA) no ano de 2010. Os profissionais foram capacitados de forma gradativa antes, durante e após a implantação da SAE, no entanto nem todos os profissionais de enfermagem passaram pelo processo de capacitação. $O$ hospital em estudo implantou parcialmente a SAE executando quatro etapas do PE: coleta de dados, diagnóstico de enfermagem, planejamento de enfermagem e implementação.

A análise de dados e a discussão dos resultados foram categorizadas de acordo com a sequência do roteiro de entrevista, agrupando-se as respostas dos profissionais de enfermagem, as quais se apresentam a seguir.

\section{Facilidades encontradas na implantação da SAE}

Quando questionados sobre as facilidades encontradas na implantação da SAE, dois enfermeiros ratificaram conhecer e compreender melhor o paciente durante o período da admissão e da hospitalização. Um dos enfermeiros destacou que a SAE direciona a atuação do mesmo com uma sequência lógica de ações, facilitando a continuidade da assistência de enfermagem. Outro enfermeiro destaca o comprometimento da equipe em desenvolver o trabalho.

A SAE proporciona uma rotina e uma sequência lógica das atividades desenvolvidas para melhorar a qualidade da assistência e compreender as necessidades do paciente. (E1)

Os sujeitos destacam que a disponibilidade de publicações e um referencial teórico, facilitou a adaptação de um instrumento de anamnese e exame físico adequado a realidade do CC na implantação da SAE.

Para os técnicos de enfermagem, as facilidades encontradas dizem respeito ao acompanhamento do paciente durante o período perioperatório até o momento da alta, a organização do trabalho e execução das atividades. De forma geral, tanto os enfermeiros quanto os técnicos de enfermagem afirmaram que a implantação da SAE traz benefícios para um atendimento ao paciente de forma holística.

Comparativamente, em estudo realizado num hospital de ensino do Rio Grande do Sul, constatou-se entre os profissionais entrevistados, que as facilidades dizem respeito à vontade dos mesmos e a possibilidade de implantar a SAE. Acreditam que o cuidado aos clientes será ampliado priorizando a individualidade e as atividades de enfermagem, por isso deve ser repensada e valorizada a aceleração do processo de implantação da SAE. ${ }^{10}$

\section{Dificuldades encontradas na implantação da SAE}

$\mathrm{Na}$ avaliação dos enfermeiros, o tempo disponível para o cumprimento da SAE e a falta de pessoal são as maiores dificuldades encontradas. Enfatizam que ocupam maior tempo do turno de trabalho com atividades administrativas. 


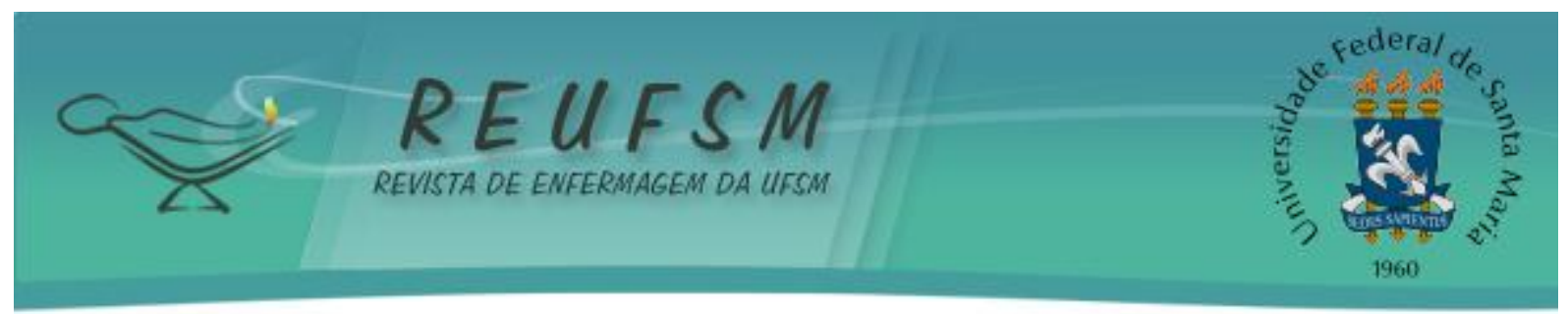

Dificuldade falta de pessoal, falta de tempo, você dedica mais tempo aos assuntos administrativos [...]. (E2)

Métodos assistenciais no período perioperatório foram definidos pela associação brasileira e internacional de enfermagem em CC, contudo encontram-se ainda em ascensão em muitos hospitais e são marcados principalmente pela falta de pessoal para prestar uma assistência qualificada aos clientes. ${ }^{7}$

Outra dificuldade apontada foi o tempo gasto na execução da SAE, a falta de prática na aplicação do PE e principalmente porque o DE não está claro para a equipe.

[...] a gente não tem prática em fazer a SAE, demora demais, é mais complicado para fazer e tem que implantar no nosso dia a dia. (E1)

Os enfermeiros destacam que para efetivar a SAE, faz-se necessário a aquisição de um sistema informatizado adequado que facilite a implementação da mesma e a otimização do tempo despendido. Hoje, a SAE é realizada manualmente com um instrumento previamente elaborado.

A SAE informatizada permite armazenar um maior número de informações e com mais qualidade nos registros, consequentemente melhora a comunicação e contribui para viabilizar a prática da enfermagem no processo de cuidar. ${ }^{11}$

Considerando o Artigo $4^{\circ}$ da Resolução no 358 de 15 de outubro de 2009 do COFEN, a SAE é uma atividade exclusiva do enfermeiro. A Resolução diz: "cabe ao enfermeiro a liderança na execução e avaliação do PE, de modo a alcançar os resultados de enfermagem esperados, cabendo-lhe, privativamente, o DE acerca das respostas da pessoa, família ou coletividade humana em um dado momento do processo saúde e doença, bem como a prescrição das ações ou intervenções de enfermagem a serem realizadas" $3: 3$

No entanto, esta é uma prática que não acontece para todos os pacientes e muitas vezes, os técnicos e secretárias desenvolvem a atividades do enfermeiro.

A entrevista [anamnese] na verdade são as secretárias que fazem, que é uma das atividades que nós deveríamos fazer [enfermeiros]. [...] se você for analisar, hoje de todos os pacientes atendidos, cerca de 60 a 70\% nós conseguimos desenvolver a SAE, o resto a gente não consegue fazer. Dá até para fazer na sala de recuperação, mas daí é só pra preencher papel. (E2)

Outro aspecto diz respeito às mudanças que geram dificuldades.

Um pouco de resistência por parte da equipe de enfermagem, que tudo que é novo tem um pouco de receio. (E4)

Para os técnicos de enfermagem, o fluxo de atividades no setor do CC dificulta um atendimento adequado e com uma sequência lógica. Muitas vezes o paciente chega no horário do procedimento e não tem tempo hábil de fazer a anamnese e exame físico, além de não ter um local adequado para atender esse paciente.

[...] é o tempo, porque às vezes o paciente chega na hora do procedimento e não dá tempo de fazer [anamnese e exame físico] $e$ também falta um local apropriado. (T2) 


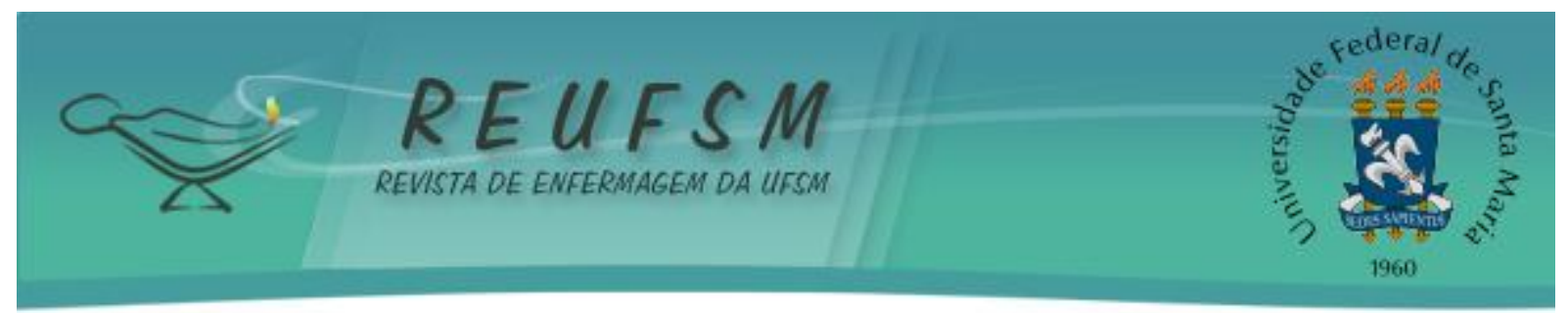

Outra dificuldade mencionada é a falta de registro da situação em que o paciente se encontra ou chega ao CC, muitas vezes não se sabe exatamente o que o paciente traz consigo (dreno ou sonda), se foi colocado no hospital ou se já vem do ambiente extra hospitalar.

Estudo revela que os registros de enfermagem integram todas as etapas e fortalecem a SAE, além de permitir uma assistência de qualidade e respaldar os profissionais legalmente. ${ }^{12}$ Resultados que nos fazem refletir acerca da importância da educação continuada e dos registros como uma forma de consolidar os cuidados prestados ao paciente.

A burocratização da SAE foi outro aspecto referido. Um técnico de enfermagem discorreu que não observa dificuldades. Para este e os demais técnicos de enfermagem, a SAE é um instrumento para checar as atividades realizadas e aprazar. O desconhecimento sobre a SAE pode prejudicar o contexto de todo o $\mathrm{PE}$, pois a maioria dos técnicos de enfermagem não sabia que os papéis que preenchem fazem parte da SAE e que para se obter um resultado satisfatório é necessário o registro de todas as etapas.

As informações analisadas pelas autoras corroboram com estudos sobre o tema, e que destacam dentre os principais fatores que dificultam a implantação da SAE: a falta de conhecimento sobre a realização do exame físico, falta de treinamento sobre o tema nas instituições de saúde, falta de registro adequado da assistência de enfermagem, conflito de papéis, dificuldade de aceitação de mudanças, falta de credibilidade nas prescrições de enfermagem, carência de pessoal, falta de estabelecimento de prioridades organizacionais, a necessidade do envolvimento das equipes e da vontade política, o ensino acadêmico, a complexidade do PE, a falta de uniformidade nas etapas e a falta de conhecimento, a educação permanente para a equipe com investimento ampliado para auxiliares e técnicos. ${ }^{10,13^{3}}$

Outro aspecto, diz respeito aos órgãos fiscalizadores que permitem o descumprimento da resolução 358/2009. O estudo finaliza, enfatizando que o preparo técnico-científico, as condições institucionais e o envolvimento de toda a equipe de enfermagem tornam-se imprescindíveis no processo de implantação e manutenção da SAE. ${ }^{13}$

\section{A implantação da SAE e o auxílio na praticidade dos serviços prestados}

Os enfermeiros mencionam que a SAE permite entender melhor o paciente, porém torna-se complexa por ser diferenciada para cada setor. Referem que a SAE proporciona segurança ao paciente e aos profissionais, possibilita um conhecimento integral das condições clinicas e psicológicas do paciente e o paciente conhece o profissional que o acompanha.

Então ela [a SAE] é fundamental, muito bom porque tu conhece o paciente, sabe o que ta acontecendo, tu acompanha, o paciente se sente mais seguro, porque tu se apresenta, tu já mostrou teu rosto pra ele $[\ldots]$... (E2)

A Resolução do COFEN n³58 de 2009, descreve que a SAE organiza o trabalho profissional quanto ao método, pessoal e instrumentos, tornando possível a operacionalização do PE. ${ }^{3}$ Para os enfermeiros do setor B, a SAE possibilitou conhecer as necessidades do paciente.

A implantação da SAE auxiliou sim, porque teu trabalho é mais prático, porque você tem uma dinâmica de ver o paciente como um todo, você faz todo o processo, através desse processo, você identifica o que o paciente precisa. É um documento que fica no 


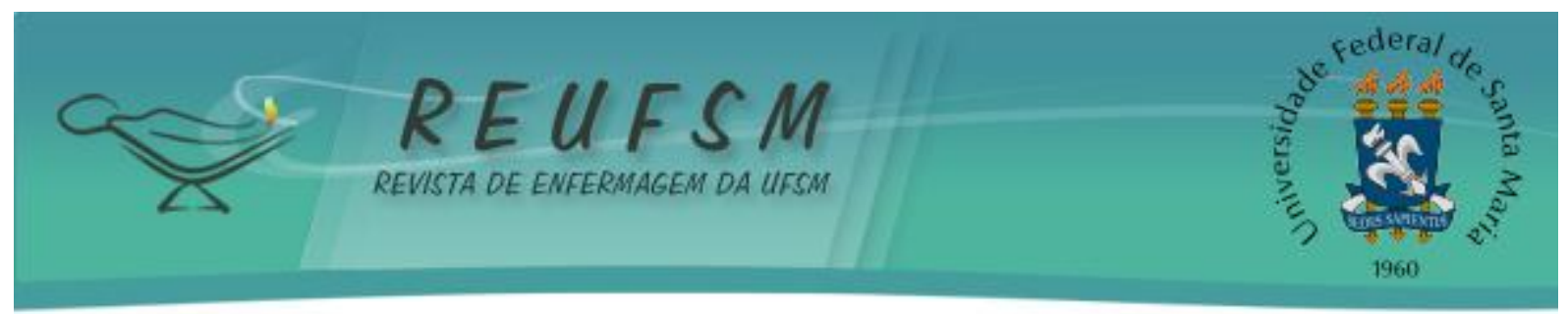

prontuário do paciente e que isso serve de base para qualquer pessoa que tiver acesso. (E4)

Para os técnicos de enfermagem, a SAE permite melhorar as anotações registradas no prontuário do paciente, tornando o serviço mais ágil.

Sim, você pode pensar que esqueceu de realizar algum cuidado. É quando você vai checar a atividade, vai ter certeza de que todos foram feitos, foram realizados. (T4)

\section{Mudanças na atuação prática do trabalho para a equipe de enfermagem}

Os enfermeiros referem que a implantação da SAE melhorou a assistência prestada ao paciente, o que antes era realizado e apenas informado verbalmente, hoje fica registrado no prontuário. A equipe se envolve mais com o paciente, houve uma melhor interação profissional $x$ paciente. Através da SAE implantaram-se novos métodos de assistência, como a escala de grau de dependência, que diminui a sobrecarga de atividades aos colaboradores.

[...] com ela [SAE] fica mais claro o cuidado, a gente consegue visualizar e entender o paciente através dos registros, mesmo sem conversar com o paciente. [...] antigamente era só verbalmente, você observava, mas não anotava. Então agora você perde um pouquinho de tempo, mas pelo menos está tudo registrado. (E1)

Aos técnicos de enfermagem, a atuação prática do serviço melhorou, as anotações de enfermagem tornaram-se mais completas, registram-se com exatidão os cuidados prestados, proporcionando o acesso às informações a todos os envolvidos.

A informatização da SAE seria um ótimo alicerce na praticidade dos serviços para a equipe de enfermagem, resultando no trabalho da enfermagem mais organizado e eficiente, facilitando os serviços e auxiliando para melhores resultados. ${ }^{14}$

\section{Mudanças na assistência ao cliente com a implantação da SAE}

Para os participantes deste estudo, o processo da SAE é trabalhoso. No setor de CC o tempo é insuficiente para realizar todas as etapas (pré, trans e pós-operatório). A carga horária alta e a falta de funcionários prejudicam a assistência prestada ao paciente, pois como o CC é uma unidade muito complexa as funções do enfermeiro se expandem da assistência para o gerenciamento. ${ }^{12}$

[...] no CC fica muito corrido, com ela [SAE] as atividades ficam muito administrativas, burocráticas e a assistência fica a desejar. (E1)

A análise das entrevistas aponta que a assistência melhorou muito, pois possibilitou conhecer o paciente e o mesmo confiar no profissional que o atende. A SAE proporciona um vínculo maior entre paciente e profissional e exige da equipe de enfermagem uma avaliação mais precisa do paciente para realizar os DE. No entanto, reconhecem que uma das limitações é não poder realizar o PE para todos os pacientes.

$O$ resultado das entrevistas corrobora com estudo realizado onde considera a SAE um instrumento que melhora a qualidade da assistência prestada. "[...] o enfermeiro precisa 


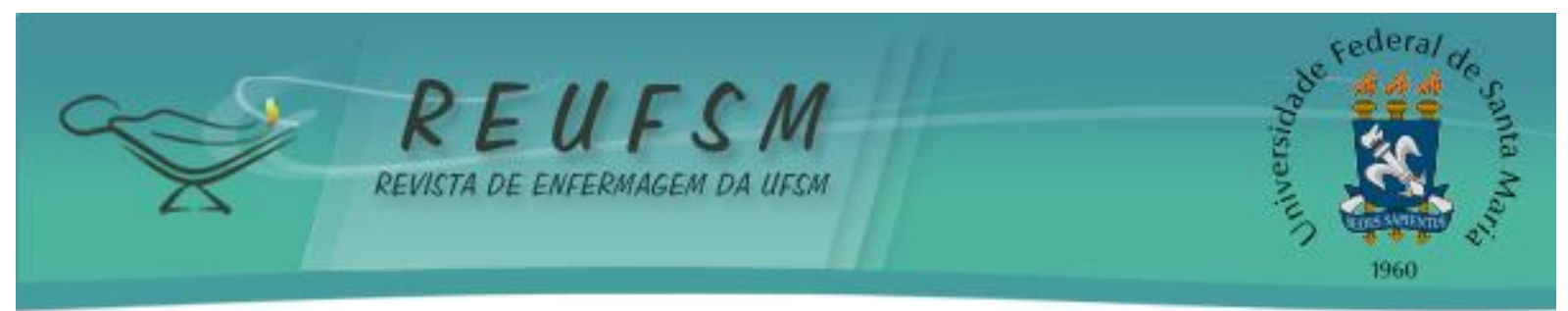

desenvolver habilidades e competências de gestão e gerência para organizar a equipe de enfermagem; o ambiente e o fluxo dos demais membros da equipe de saúde". 15:315

Para os técnicos de enfermagem a interação com o paciente melhorou e se obtém o conhecimento completo do paciente quando se realiza a anamnese no período préoperatório. A organização das atividades melhorou, os cuidados prestados devem ser checados e não são deixados de realizar. É importante ressaltar que, para alguns dos técnicos de enfermagem, a SAE não passa de uma rotina e que antes da implantação também era realizada, no entanto, agora é mais específica, com mais detalhes a respeito do paciente e com registro no prontuário do mesmo.

\section{Valorização e autonomia profissional da equipe de enfermagem}

Quando indagados sobre a percepção da valorização e autonomia do enfermeiro com a implantação da SAE, as respostas foram otimistas, criaram-se rotinas que antes não existiam, demais profissionais passaram a conhecer e reconhecer o trabalho da enfermagem e a utilizar os registros no prontuário do paciente para a assistência.

A SAE "constitui-se um instrumento de fundamental importância para que o enfermeiro possa gerenciar e otimizar a assistência de enfermagem de forma organizada, segura, dinâmica e competente". 16:3

No discurso dos enfermeiros, a valorização da equipe de enfermagem num contexto geral, faz com que o paciente sinta-se valorizado indiretamente.

[...] ficam mais responsabilidades, porque dei meu nome, to usando o meu crachá, ele [o paciente] vai me conhecer, vai saber quem eu sou, vai ver como eu to atendendo. (E2)

A SAE organiza a equipe, faz planejar as suas atividades, fazer com que o setor melhore, quem vai sair mais satisfeito na verdade é o cliente. Perante a empresa, a auditoria, a equipe está fazendo um trabalho, e se formos reconhecidos por isso, com certeza é um meio de valorização profissional. (E4)

Os técnicos de enfermagem sentem-se mais valorizados porque a SAE permite conhecer melhor o paciente e informar aos demais profissionais a historicidade do mesmo de uma forma clara e verdadeira, resultando em melhor comunicação entre a equipe multiprofissional. Sentem-se mais valorizados pelo próprio paciente a partir dos serviços prestados aos mesmos.

"O profissional de enfermagem, através do conhecimento e operacionalização da SAE, representa um importante elemento articulador e mobilizador do processo de melhoria no contexto interdisciplinar da saúde". 17:6

\section{Visibilidade da Enfermagem com a implantação da SAE}

Ao serem questionados, os enfermeiros do CC afirmam que a SAE permitiu estarem mais presentes nas salas cirúrgicas, melhorando a assistência pelo conhecimento ampliado em relação ao paciente. Porém, não depende somente da SAE para se tornar visível, se o profissional concentrar suas atividades na gestão, se torna ausente na assistência. Os resultados da pesquisa apontam uma relação direta da SAE como um documento e não como processo de trabalho em enfermagem. 


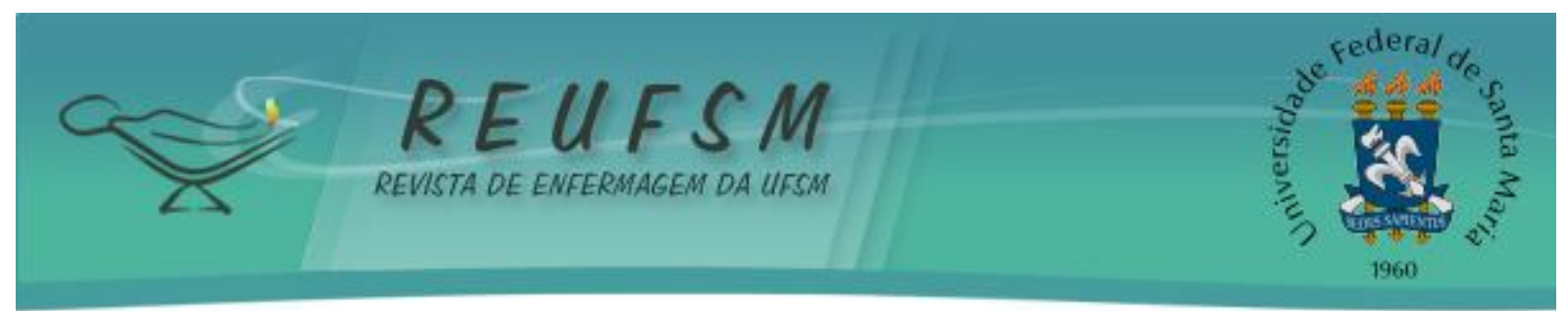

A visibilidade profissional constrói-se, nesse sentido, a partir das atitudes individuais que formam o coletivo, e que, por sua vez, se refletem nos diferentes espaços e campos de atuação profissional. ${ }^{17}$

Os enfermeiros relatam que a SAE no CC se torna muito repetitiva, porque os questionamento realizadas na anamnese, no período pré-operatório, também são feitos pelos anestesistas na sala de operação. Os enfermeiros sugerem a adoção de um instrumento que abrangesse toda a equipe multiprofissional, e que não se torne incômodo ao paciente.

Um enfermeiro destaca a importância da SAE. Em hospitais que não têm a SAE implantada muitos pacientes recebem alta hospitalar sem saber o nome da pessoa que o cuidou, destaca que a SAE proporciona essa visibilidade e faz com que a equipe se comprometa e se dedique mais aos pacientes.

[...] antigamente eu nem via o paciente, tu ia lá pra passar sonda, para puncionar e saia, ou conferir uma caixa, essas coisas mais burocráticas mesmo. Com a SAE apesar de ser burocrática, faz com que esteja na sala de cirurgia e acompanhe o procedimento, acompanhar no pré, no trans e no pós-operatório, o que vai precisar no pós, alguns cuidados específicos que vai precisar. (E1)

Para os enfermeiros do setor $B$, a SAE é um trabalho diferenciado, a equipe de enfermagem destaca-se e com a certificação da ONA o retorno do trabalho realizado foi positivo. Para os técnicos de enfermagem, a categoria tornou-se mais visível pela organização dos serviços e pela exatidão dos registros de enfermagem.

[...] se você faz alguma coisa que não é no papel você não é vista. Eu acredito que com a SAE é bem mais visível, nosso cuidado, nossa assistência. (T4)

É essencial que os próprios profissionais se responsabilizem pelas suas ações, se desacomodem e enfrentem os desafios, procurando apropriar-se das suas competências, de modo a intervir de forma pró-ativa nas diferentes demandas sociais. ${ }^{17}$

\section{CONCLUSÃO}

Os resultados da pesquisa apontam que a implantação da SAE acarretou progressos em relação a: segurança do paciente; possibilidade de conhecer o paciente de forma holística; os registros de enfermagem tornaram-se mais precisos facilitando o acesso às informações do paciente; organização e sequência das atividades de enfermagem e a continuidade da assistência de enfermagem.

A SAE não é uma tarefa fácil para o enfermeiro e para a equipe de enfermagem. A falta do planejamento para a implantação, execução e a falta do entendimento sobre o que é a SAE ficam evidentes, sobretudo para os técnicos de enfermagem.

0 medo em participar do estudo limitou a amostragem, no entanto foi possível identificar a necessidade de um programa de educação continuada junto à equipe de enfermagem e equipe multiprofissional da instituição, bem como a implantação de um grupo de estudos que viabilizem a correção deste déficit.

Percebeu-se a necessidade de implantar um sistema informatizado para aprimorar o processo de trabalho, otimizando o tempo e a organização dos serviços. 


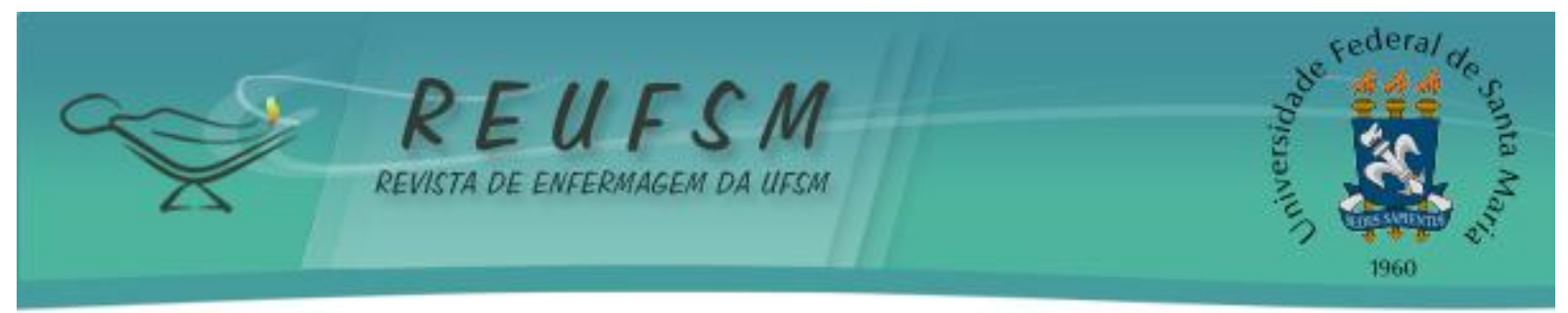

Os achados apontam a necessidade de estudos que fortaleçam a implantação e implementação da SAE e uma reflexão sobre o dimensionamento de pessoal sob a perspectiva de viabilizar estratégias que culminem para uma SAE efetiva e com resultados satisfatórios à equipe de enfermagem, aos pacientes, aos familiares, à comunidade e à própria instituição.

\section{REFERÊNCIAS}

1. Aguilar VM, Robles ALM. Processo de enfermagem: modelo de interação terapêutica e uso das linguagens NANDA, CIE (NIC), CRE (NOC); [tradução Verônica Calbo]. São Paulo: DCL; 2009.

2. Tannure MC, Gonçalves AMP. SAE, Sistematização da Assistência de Enfermagem: guia prático. Rio de Janeiro: Guanabara Koogan; 2009.

3. Conselho Federal de Enfermagem. Resolução COFEN n 358/2009. [acesso em $2011 \mathrm{fev}$ 24]. Disponível em: http://site.portalcofen.gov.br/node/4309.

4. Malagutti W, Bonfim IM. Enfermagem no centro cirúrgico: atualidades e perspectivas no ambiente cirúrgico. São Paulo: Martinari; 2009.

5. Fonseca RMP, Peniche ACG. Enfermagem em centro cirúrgico: trinta anos após criação do Sistema de Assistência de Enfermagem Perioperatória. Acta Paul Enferm. 2009;22(4):428-33.

6. Associação Brasileira de Enfermeiros de Centro Cirúrgico, Recuperação Anestésica e Centro de Material e Esterilização. SOBECC. São Paulo. [acesso em 2011 abr 2]. Disponível em: http: //portal.sobecc.org.br/?p=209.

7. Carvalho R, Bianchi ERF. Enfermagem em centro cirúrgico e recuperação. Barueri, SP: Manole; 2007.

8. Alfaro-Lefevre R. Aplicação do processo de enfermagem: um guia passo a passo. $4^{\mathrm{a}}$ ed. Porto Alegre: Artes Médicas; 2000.

9. Bardin L. Análise de conteúdo. Rio de Janeiro: Edições 70; 2000.

10. Luiz FF, Padoin SMM, Neves ET, Ribeiro AC, Tronco CS. A sistematização da assistência de enfermagem na perspectiva da equipe de um hospital de ensino. Rev Eletrônica Enferm. 2010:12(Spec):655-9.

11. Almeida DMBM. Sistematização da assistência de enfermagem informatizada em unidade de cuidado semi-intensivo [dissertação]. São Paulo: USP; 2011. 192 p.

12. Azevêdo LMN, Oliveira AG, Malveira FAS, Valença CN, Costa EO, Germano RM. A visão da equipe de enfermagem sobre seus registros. Rev RENE. 2012;13(1):64-73.

13. Cavalcante RB, Otoni A, Bernardes MFVG, Cunha SGS, Santos CS, Silva PC. Experiências de sistematização da assistência de enfermagem no Brasil: um estudo bibliográfico. Rev Enferm UFSM. [internet]. 2011 [acesso em 2012 fev 26] set/dez;1(3):461-71. Disponível em: http://cascavel.ufsm.br/revistas/ojs-

\subsection{2/index.php/reufsm/article/view/2832/2396}

14. Oliveira M. Gerenciamento de novas tecnologias em Centro Cirúrgico pelas enfermeiras nos hospitais de Feira de Santana- Bahia. Rev Bras Enferm. 2004;57(3):292-7.

15. Krauzer IM, Gelbcke FL. Sistematização da assistência de enfermagem: potencialidades reconhecidas pelos enfermeiros de um hospital público. Rev Enferm UFSM. [internet] 2011 


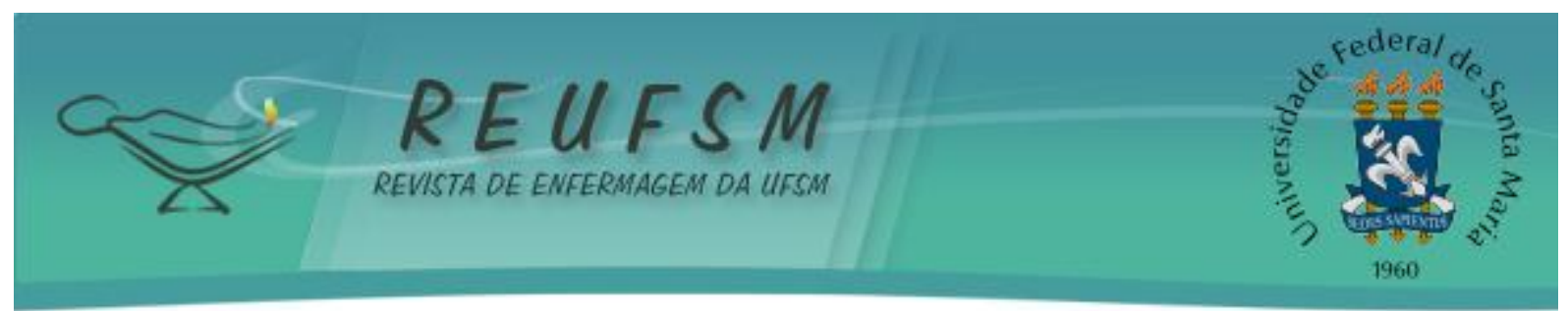

[acesso em 2012 fev 26] set/dez;1(3): 308-17. Disponível em:

http://cascavel.ufsm.br/revistas/ojs-2.2.2/index.php/reufsm/article/view/3592/2379

16. Backes DS, Schwartz E. Implementação da sistematização da assistência de enfermagem: desafios e conquistas do ponto de vista gerencial. Ciênc Cuid Saúde. 2005 maio/ago;4(2):182-8.

17. Erdmann AL, Fernandes JV, Melo C, Carvalho BR, Menezes Q, Freitas R, et al. A visibilidade da profissão de enfermeiro: reconhecendo conquistas e lacunas. Rev Bras Enferm. 2009;62(4):637-43.

Data de recebimento: $12 / 04 / 2012$

Data de aceite: 08/07/2012

Contato com autor responsável: Edlamar Kátia Adamy

Endereço: Rua Claudio Stakonski, 90D, Apto 203B. Chapecó, SC.

CEP: 89806-154

E-mail: katiadamy@hotmail.com 